\title{
Stability Condition of State-Delayed Systems Based on Stability-Guaranteed Discretization ${ }^{\dagger}$
}

\author{
Young Soo SuH* and Seiichi SHIN*
}

\begin{abstract}
This paper is concerned with a new delay-dependent stability condition for state-delayed systems. This stability condition is based on discretization of the infinite-dimensional kernels of state-delayed systems. The discretized system of a state-delay system is not finite dimensional; thus the stability condition cannot be derived by simple discretization. In this paper, the discretized system is divided into a finite-dimensional part and an error part. The stability condition is derived based on the finite-dimensional part explicitly taking account of the error part. The lifting technique that is used in sampled-data control is used to derive the stability condition. The stability condition is given in the form of an $H_{\infty}$ norm test for discrete-time systems. The stability condition is shown to converge to the exact stability condition as the order of discretization approaches infinity.
\end{abstract}

Key Words: time-delay system, state-delayed system, lifting technique, infinite-dimensional system, approximation, robust control

\section{Introduction}

There are several forms of necessary and sufficient stability conditions for state-delayed systems ${ }^{1)}$. Most of these conditions are expressed in terms of quasipolynomials or operator Lyapunov equations, which are not easy to compute ${ }^{2)}$. Hence several sufficient stability conditions, which are easier to compute, are proposed by many authors: delay-independent stability conditions $3), 4)$ and delay-dependent stability conditions ${ }^{5), 6)}$. A choice of these stability conditions depends on a trade-off between computational burden and conservatism: there is a general tendency that stability conditions are less conservative if they require more computation.

In this paper, we propose a new delay-dependent stability condition, which has two desirable features. One is that the stability condition provides an explicit method to make a trade-off between computational burden and conservatism. The other one is that the stability condition is applicable to synthesis problems.

Our new stability condition is based on discretization of state-delayed systems, where the discretized system is divided into a finite-dimensional part and a discretization error part. The stability condition consists of two conditions. The first condition is for checking the stability

\footnotetext{
$\dagger$ Presented in the 24th SICE Symposium on Control Theory (1995.6)

* Faculty of Engineering, The University of Tokyo, Bunkyo$\mathrm{ku}$, Tokyo

(Received March 13, 1996)

(Revised December 11, 1996)
}

of the finite-dimensional part of the discretized system, where the order of discretization can serve as a parameter to make a trade-off between computational burden and conservatism. The effect of the discretization error is considered in the second condition. This explicit treatment of the discretization error is a new approach to stability analysis of state-delayed systems and is the main contribution of this paper. The discretization error is treated using the lifting technique ${ }^{8) \sim 10)}$ and the second condition is given in the form of an $H_{\infty}$ norm test for discrete-time systems ${ }^{11)}$. Due to this fact, our condition can be easily applicable to systhesis problems, too. Finally it is shown that our stability condition converges to the exact stability condition as the order of discretization approaches infinity

Notation: Let $L_{n}^{2}[0, a)$ denote the set of Lebesque square integrable signals, where signals are $n$-dimensional vectors of scalar signals. Let $l_{R^{n}}$ and $l_{L_{n}^{2}[0, a)}$ denote the set of square summable sequences on $R^{n}$ and $L_{n}^{2}[0, a)$, respectively. The lifting operator $W_{\tau}: L_{n}^{2}[0, \infty) \rightarrow l_{L_{n}^{2}[0, \tau)}$ 8) is defined by $\hat{f}=W_{\tau} f, \hat{f}_{k}(r)=f(\tau k+r), 0 \leq r<$ $\tau, k=0,1, \cdots$ Let $\|\cdot\|_{2}$ denote the standard Euclidean norm of vectors. Let $\|\cdot\|_{L_{n}^{2}[0, a)}$ be defined by $\|f(t+r)\|_{L_{n}^{2}[0, a)}:=\left(\int_{0}^{a}\|f(t+r)\|_{2}^{2} d r\right)^{\frac{1}{2}}, 0 \leq r<a$. Both the induced norm from $l_{L_{a}^{2}[0, \tau)}$ to $l_{L_{b}^{2}[0, \tau)}$ and the induced norm from $l_{R^{a}}$ to $l_{R^{b}}$ are denoted by the same symbol $\|\cdot\|_{\infty}$. For a matrix or an operator $M, \rho(M)$ denotes the spectral radius of $M$. The upper linear fractional transformation of systems $Q$ and $R$ is denoted by $\operatorname{LFT}(Q, R):=Q_{22}+Q_{21} R\left(I-Q_{11} R\right)^{-1} Q_{12}$ where 


$$
Q=\left[\begin{array}{ll}
Q_{11} & Q_{12} \\
Q_{21} & Q_{22}
\end{array}\right]
$$

\section{Main Result}

Let us consider the following state-delayed system

$$
G: \begin{aligned}
\dot{x}(t) & =A_{0} x(t)+A_{1} x(t-h)+B u(t) \\
y(t) & =C x(t),
\end{aligned}
$$

whose characteristic equation is given by

$$
\operatorname{det}\left(s I-A_{0}-A_{1} e^{-s h}\right)=0
$$

where $x(t) \in R^{n}, u(t) \in R^{r}$, and $y(t) \in R^{p}$. Let $x(r):=\phi(r) \in L_{n}^{2}[-h, 0],-h \leq r \leq 0$ be the initial states of $G$.

A new sufficient stability condition (exponential stability) is derived using discretization of the system (1). By exponential stability of $G$, we mean

$$
\|x(t)\|_{2} \leq M e^{-\alpha t}\|\phi(r)\|_{L_{n}^{2}[-h, 0]}, \quad-h \leq r \leq 0, \quad t \geq 0,
$$

for some $0 \leq M<\infty$ and $\alpha>0$.

Unlike a non-delayed system or an input-delayed system, discretization of a state-delayed system cannot be expressed in terms of a finite-dimensional discrete-time system. To see this, discretize the system (1) with $\tau=h / N$, where $h$ is the delay time in the state of (1) and $N$ is a positive integer:

$$
\begin{aligned}
& x(k \tau+\tau)= \\
& \quad e^{A_{0} \tau} x(k \tau)+\int_{0}^{\tau} e^{A_{0}(\tau-r)} A_{1} x(k \tau-N \tau+r) d r \\
& \quad+\int_{0}^{\tau} e^{A_{0}(\tau-r)} B u(k \tau+r) d r .
\end{aligned}
$$

Due to the first integral term, the above equation cannot be expressed in terms of a finite-dimensional discrete-time system. To overcome this problem, we will artificially divide the above equation into a finite-dimensional discretetime part and a discretization error part, just by adding the term $\int_{0}^{\tau} e^{A_{0}(\tau-r)} A_{1} x(k \tau-N \tau) d r$ and subtracting it as follows:

$$
\begin{aligned}
& x(k \tau+\tau)= \\
& \quad\left[e^{A_{0} \tau} x(k \tau)+\int_{0}^{\tau} e^{A_{0}(\tau-r)} A_{1} d r x(k \tau-N \tau)\right. \\
& \left.\quad+\int_{0}^{\tau} e^{A_{0}(\tau-r)} B u(k \tau+r) d r\right]_{1} \\
& +\left[\int_{0}^{\tau} e^{A_{0}(\tau-r)} A_{1}\{x(k \tau-N \tau+r)\right. \\
& \quad-x(k \tau-N \tau)\} d r]_{2}, \quad-\tau \leq r<0 .
\end{aligned}
$$

The part $[\cdot]_{\mathbf{1}}$ will be regarded as the finite-dimensional discrete-time part and $x(k \tau-N \tau+r)-x(k \tau-N \tau)$ as the discretization error. We will show that the state-delayed system (1) is stable if the discretized part [ $]_{\mathbf{1}}$ is stable and the discretization error part $[\cdot]_{2}$ satisfies some condition.

A stability condition could be derived if the magnitude of the discretization error is known. It is, however, difficult to estimate the magnitude of the discretization error.
We will use the following property of the discretization error instead of estimating the magnitude of the discretization error. We note that the discretization error at $k$, $x(k \tau+t)-x(k \tau)$, can be expressed by the part $[\cdot]_{\mathbf{1}}$ and the discretization error at $k-N, x((k-N) \tau+t)-x((k-N) \tau)$ :

$$
\begin{aligned}
& x(k \tau+t)-x(k \tau)= \\
& \left(e^{A_{0} t}-I\right) x(k \tau)+\int_{0}^{t} e^{A_{0}(t-r)} A_{1} d r x(k \tau-N \tau) \\
& +\int_{0}^{t} e^{A_{0}(t-r)} B u(k \tau+r) d r \\
& +\int_{0}^{t} e^{A_{0}(t-r)} A_{1}\{x((k-N) \tau+r) \\
& -x((k-N) \tau)\} d r .
\end{aligned}
$$

If we write $\hat{v}_{k}(t)=x(k \tau+t)-x(k \tau)$ and $\hat{r}_{k}(t)=$ $x((k-N) \tau+t)-x((k-N) \tau)$, we have

$$
\hat{r}_{k}(t)=\hat{v}_{k-N}(t) .
$$

In order to obtain simpler representation, here we use the lifting technique ${ }^{8)}$. Using (4) and (5), we introduce $\hat{G}_{N}$ and $\hat{\Delta}$, which can be considered as a lifted system of $G$ i.e., $\operatorname{LFT}\left(\hat{G}_{N}, \hat{\Delta}\right)=W_{\tau}^{-1} G W_{\tau}$ (see Fig. 1).

$$
\begin{gathered}
\hat{G}_{N}=\left[\begin{array}{ll}
\hat{G}_{N 11} & \hat{G}_{N 12} \\
\hat{G}_{N 21} & \hat{G}_{N 22}
\end{array}\right]: \\
{\left[\begin{array}{c}
l_{L_{n}^{2}[0, \tau)} \\
l_{L_{r}^{2}[0, \tau)}
\end{array}\right] \rightarrow\left[\begin{array}{c}
l_{L_{n}^{2}[0, \tau)} \\
l_{L_{p}^{2}[0, \tau)}
\end{array}\right],}
\end{gathered}
$$

whose realization is given by

$$
\begin{aligned}
& \hat{X}_{k+1}=\hat{A}_{N} \hat{X}_{k}+\hat{B}_{1} \hat{r}_{k}(s)+\hat{B}_{2} \hat{u}_{k}(s) \\
& \hat{v}_{k}(t)=\hat{C}_{1} \hat{X}_{k}+\hat{D}_{11} \hat{r}_{k}(s)+\hat{D}_{12} \hat{u}_{k}(s) \\
& \hat{y}_{k}(t)=\hat{C}_{2} \hat{X}_{k}+\hat{D}_{21} \hat{r}_{k}(s)+\hat{D}_{22} \hat{u}_{k}(s) \\
& \hat{r}_{k}(t)=\hat{\Delta} \hat{v}_{k}(s),
\end{aligned}
$$

where

$$
\hat{A}_{N}=\left[\begin{array}{ccccc}
e^{A_{0} \tau} & 0 & \cdots & & \int_{0}^{\tau} e^{A_{0} r} A_{1} d r \\
I & 0 & & & \\
0 & I & & & \\
\vdots & & \ddots & & \\
0 & & & I &
\end{array}\right],
$$$$
\hat{B}_{1}(s)=\left[\begin{array}{c}
e^{A_{0}(\tau-s)} A_{1} \\
0 \\
\vdots \\
0
\end{array}\right], \hat{B}_{2}(s)=\left[\begin{array}{c}
e^{A_{0}(\tau-s)} B \\
0 \\
\vdots \\
0
\end{array}\right]
$$$$
\hat{C}_{1}(t)=\left[\begin{array}{lll}
\left(e^{A_{0} t}-I\right) & 0 \cdots 0 & \int_{0}^{t} e^{A_{0} r} d r A_{1}
\end{array}\right],
$$$$
\hat{C}_{2}(t)=\left[\begin{array}{llll}
C e^{A_{0} t} & 0 \cdots 0 & C \int_{0}^{t} e^{A_{0} r} d r A_{1}
\end{array}\right],
$$

$$
\begin{aligned}
& \hat{D}_{11}(t, s)=e^{A_{0}(t-s)} A_{1} \mathbf{1}(t-s), \\
& \hat{D}_{12}(t, s)=e^{A_{0}(t-s)} B \mathbf{1}(t-s), \\
& \hat{D}_{21}(t, s)=C e^{A_{0}(t-s)} A_{1} \mathbf{1}(t-s), \\
& \hat{D}_{22}(t, s)=C e^{A_{0}(t-s)} B \mathbf{1}(t-s) .
\end{aligned}
$$


The same symbol is used for an operator and its kernel function, for example $\hat{D}_{11}(t, s)$ refers to the kernel function representing the operator $\hat{D}_{11}$. The operator $\hat{A}_{N}$ is an $(N+1) \times(N+1)$ matrix of operators, whose elements are $R^{n} \rightarrow R^{n}$. The operators $\hat{B}_{1}$ and $\hat{B}_{2}$ are $(N+1) \times 1$ matrices of operators, whose elements are $l_{L_{n}^{2}[0, \tau)} \rightarrow R^{n}$ and $l_{L_{n}^{2}[0, \tau)} \rightarrow R^{n}$, respectively. The operators $\hat{C}_{1}$ and $\hat{C}_{2}$ are $1 \times(N+1)$ matrices of operators, whose elements are $R^{n} \rightarrow l_{L_{n}^{2}[0, \tau)}$ and $R^{n} \rightarrow l_{L_{p}^{2}[0, \tau)}$, respectively. The $\mathbf{1}(t-s)$ is defined by $\mathbf{1}(t-s)=1, t-s \geq 0$ and $\mathbf{1}(t-s)=0, t-s<0$.

The relation (5) is realized by introducing an $N$-step delay system $\hat{\Delta}$. The delay system $\hat{\Delta}$ is defined by $\hat{\Delta}: l_{L_{n}^{2}[0, \tau)} \rightarrow l_{L_{n}^{2}[0, \tau)}, \quad \hat{\Delta} \hat{f}_{k}=\hat{f}_{k-N}$, whose realization is given by

$$
\begin{aligned}
& \hat{Y}_{k+1}=\hat{A}_{\hat{\Delta}} \hat{Y}_{k}+\hat{B}_{\hat{\Delta}} \hat{v}_{k}(s) \\
& \hat{r}_{k}(t)=\hat{C}_{\hat{\Delta}} \hat{Y}_{k},
\end{aligned}
$$

where

$$
\begin{aligned}
& \hat{A}_{\hat{\Delta}}=\left[\begin{array}{cccc} 
& I & & \\
& & \ddots & \\
& & & I \\
& & &
\end{array}\right], \hat{B}_{\hat{\Delta}}=\left[\begin{array}{c}
0 \\
\vdots \\
0 \\
I
\end{array}\right], \\
& \hat{C}_{\hat{\Delta}}=\left[\begin{array}{llll}
I & 0 & \cdots & 0
\end{array}\right] .
\end{aligned}
$$

The operator $\hat{A}_{\hat{\Delta}}$ is an $N \times N$ matrix of operators, whose element are $l_{L_{n}^{2}[0, \tau)} \rightarrow l_{L_{n}^{2}[0, \tau)}$. The operator $\hat{B}_{\hat{\Delta}}$ is an $N \times 1$ matrix of operators, whose elements are $l_{L_{n}^{2}[0, \tau)} \rightarrow$ $l_{L_{n}^{2}[0, \tau)}$. The operator $\hat{C}_{\hat{\Delta}}$ is a $1 \times N$ matrix of operators, whose elements are $l_{L_{n}^{2}[0, \tau)} \rightarrow l_{L_{n}^{2}[0, \tau)}$. The operator $I$ is an identity operator on $l_{L_{n}^{2}[0, \tau)}$.

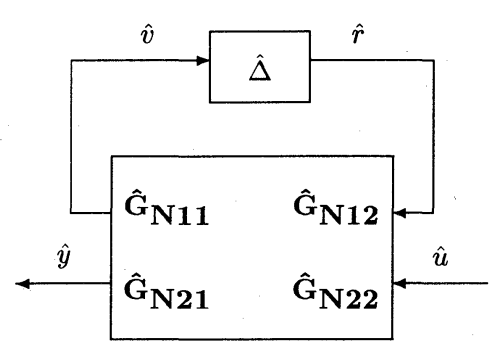

Fig. $1 \operatorname{LFT}\left(\hat{G}_{N}, \hat{\Delta}\right)$

The $\hat{G}_{N 22}$ is the finite-dimensional discrete-time part of $G$ and the $N$-step delay system $\hat{\Delta}$, which is a normbounded system, represents the discretization error part. If there is no state-delayed element, $\hat{\Delta}$ term is not necessary, and $\hat{y}$ and $\hat{u}$ in Fig. 1 are just the lifted signals of $y$ and $u$ of the system $G$, respectively. Now we begin our in- vestigation by clarifying relationship between the system $\hat{G}_{N}$ (with $\hat{\Delta}$ ) and the original system $G$.

Lemma 1. Let $\phi(r) \in L_{n}^{2}[-h, 0],-h \leq r \leq 0$ be the initial states of $G$ and $u(t), t \geq 0$ be the input to $G$. Given the initial conditions $x(r)=\phi(r),-h \leq r \leq 0$ and the input $u(t)$, let $x(t)$ and $y(t)$ be the state and the output of the system $G$, respectively. If the initial states of $\hat{G}_{N}$ and $\hat{\Delta}$ are given by

$$
\begin{aligned}
\hat{X}_{0}= & {\left[\phi^{\prime}(0), \quad \cdots, \quad \phi^{\prime}(-N \tau)\right]^{\prime}, } \\
\hat{Y}_{0}=\quad\left[\phi^{\prime}(-\tau+r)-\phi^{\prime}(-\tau),\right. & \\
& \left.\cdots, \quad \phi^{\prime}(-N \tau+r)-\phi^{\prime}(-N \tau)\right]^{\prime}, \\
& 0 \leq r<\tau,
\end{aligned}
$$

and $\hat{u}=W_{\tau} u$, then we have $\hat{X}_{k}=\left[x^{\prime}(k \tau), \cdots, x^{\prime}((k-\right.$ $N) \tau)]^{\prime}, k \geq 1$ and $\hat{y}=W_{\tau} y$. Moreover, there holds $\hat{v}_{k}(t)=\hat{r}_{k+N}(t)=x(k \tau+t)-x(k \tau), 0 \leq t<\tau, k \geq 0$.

Proof. Note that the state of the system $G$ is generated by the following:

$$
\begin{aligned}
& x(k \tau+t)= \\
& e^{A_{0} t} x(k \tau)+\int_{0}^{t} e^{A_{0}(t-r)} A_{1} d r x(k \tau-N \tau) \\
& +\int_{0}^{t} e^{A_{0}(t-r)} B u(k \tau+r) d r \\
& +\int_{0}^{t} e^{A_{0}(t-r)} A_{1}\{x(k \tau-N \tau+r) \\
& \quad-x(k \tau-N \tau)\} d r .
\end{aligned}
$$

The arguments can be verified by comparing (8) with (6) and (7).

Lemma 1 is, in a sense, a restatement of the fact that the state of a state-delayed system can be completely determined if the initial conditions $\phi(r),-h \leq r \leq 0$ are given. Now we derive a condition for the exponential stability of the system $\hat{G}_{N}$ with $\hat{\Delta}$. By exponential stability of $\hat{G}_{N}$ with $\hat{\Delta}$, we mean

$$
\left\|\hat{X}_{k}\right\|_{2} \leq M \beta^{k}\left(\left\|X_{0}\right\|_{2}+\left\|Y_{0}\right\|\right), \quad k \geq 0
$$

for some $0<M<\infty, 0 \leq \beta<1$ and

$$
\left\|Y_{0}\right\|:=\sum_{i=1}^{N}\|\phi(-i \tau+r)-\phi(-i \tau)\|_{L_{n}^{2}[0, \tau)}, 0 \leq r<\tau .
$$

Lemma 2. If $\rho\left(\hat{A}_{N}\right)<1$ and $\left\|\hat{G}_{N 11}\right\|_{\infty}<1$, then the system $\hat{G}_{N}$ with $\hat{\Delta}$ is exponentially stable.

Proof. From (6) and (7), we have

$$
\begin{aligned}
\hat{X}_{k}= & \hat{A}_{N}^{k} \hat{X}_{0}+\sum_{i=0}^{N-1} \hat{A}_{N}^{k-1-i} \hat{B}_{1} \hat{r}_{i} \\
& +\sum_{i=N}^{k-1} \hat{A}_{N}^{k-1-i} \hat{B}_{1} \hat{v}_{i-N}, \quad k \geq N+1 .
\end{aligned}
$$

Throughout the proof, let $M_{i}$ and $\beta_{i}$ be $0 \leq M_{i}<\infty$ and $0 \leq \beta_{i}<1$ for all $i$, respectively. Since $\rho\left(\hat{A}_{N}\right)<1$, we obtain $(k \geq N+1)$ 


$$
\begin{aligned}
\left\|\hat{X}_{k}\right\|_{2} \leq \quad & M_{1} \beta_{1}^{k}\left\|\hat{X}_{0}\right\|_{2} \\
& +M_{2} \sum_{i=0}^{N-1} \beta_{1}^{k-1-i}\left\|\hat{r}_{i}\right\|_{L_{n}^{2}[0, \tau)} \\
& +\sum_{i=N}^{k-1} M_{3} \beta_{1}^{k-1-i}\left\|\hat{v}_{i-N}\right\|_{L_{n}^{2}[0, \tau)} \\
\leq \quad & M_{4} \beta_{2}^{k}\left(\left\|\hat{X}_{0}\right\|_{2}+\left\|\hat{Y}_{0}\right\|\right) \\
& +\sum_{i=N}^{k-1} M_{3} \beta_{1}^{k-1-i}\left\|\hat{v}_{i-N}\right\|_{L_{n}^{2}[0, \tau)}
\end{aligned}
$$

In the above, the relation $\hat{Y}_{0}=\left[\hat{r}_{0}, \cdots, \hat{r}_{N-1}\right]$ is used. With the following condition, which will be proved later,

$$
\left\|\hat{v}_{i-N}\right\|_{L_{n}^{2}[0, \tau)} \leq M_{5} \beta_{3}^{i}\left(\left\|\hat{X}_{0}\right\|_{2}+\left\|\hat{Y}_{0}\right\|\right)
$$

we have

$$
\begin{aligned}
\left\|\hat{X}_{k}\right\|_{2} \leq & M_{4} \beta_{2}^{k}\left(\left\|\hat{X}_{0}\right\|_{2}+\left\|\hat{Y}_{0}\right\|\right) \\
& +\sum_{i=N}^{k-1} M_{3} \beta_{1}^{k-1-i} M_{5} \beta_{3}^{i} \\
\leq & M_{4} \beta_{2}^{k}\left(\left\|\hat{X}_{0}\right\|_{2}+\left\|\hat{Y}_{0}\right\|\right)+M_{6} \beta_{4}^{k} \\
\leq & M_{7} \beta_{5}^{k}\left(\left\|\hat{X}_{0}\right\|_{2}+\left\|\hat{Y}_{0}\right\|\right), k \geq N+1 .
\end{aligned}
$$

The proof is completed if we show (10). From (6) and (7), we have

$$
\hat{v}=\hat{G}_{N} \hat{\Delta} \hat{v}+\hat{p}+\hat{q},
$$

where $\hat{G}_{N} \hat{\Delta} \hat{v}$ denotes the output of $\hat{G}_{N} \hat{\Delta}$ with zero initial states, and $\hat{p} \in l_{L_{n}^{2}[0, \tau)}$ and $\hat{q} \in l_{L_{n}^{2}[0, \tau)}$ denote

$$
\begin{aligned}
& \hat{p}_{k}:= \begin{cases}\hat{r}_{k} & 0 \leq k \leq N-1 \\
0 & k>N\end{cases} \\
& \hat{q}_{k}:=\hat{C}_{1} \hat{A}_{N}^{k-1} \hat{X}_{0} \quad k \geq 0,
\end{aligned}
$$

respectively. We note that $\left(I-\hat{G}_{N} \hat{\Delta}\right)^{-1}$ exists and is bounded (Theorem 6.7.1 ${ }^{15)}$ ) since

$$
\left\|\hat{G}_{N} \hat{\Delta}\right\|_{\infty} \leq\left\|\hat{G}_{N}\right\|_{\infty}\|\hat{\Delta}\|_{\infty} \leq\left\|\hat{G}_{N}\right\|_{\infty}<1 .
$$

We can express $\hat{y}=\left(I-\hat{G}_{N} \hat{\Delta}\right)^{-1} \hat{u}$ as

$$
\begin{aligned}
z_{k+1} & =\hat{A} z_{k}+\hat{B} \hat{u}_{k} \\
\hat{y}_{k} & =\hat{C} z_{k}+\hat{D} \hat{u}_{k},
\end{aligned}
$$

where $(\hat{A}, \hat{B}, \hat{C}, \hat{D})$ are all bounded operators and can be computed from (6) and (7). We note that the state space of (13) is not finite-dimensional; thus $\hat{A}$ is an operator. Introducing the $z$-transform ${ }^{10)}$

$$
(Z \hat{u})(z):=\sum_{k=0}^{\infty} \hat{u}_{k} z^{k}
$$

where $\hat{u} \in l_{L_{n}^{2}[0, \tau)}$, we define the $z$-transform of (13) as

$$
\left(I-\hat{G}_{N}(z) \hat{\Delta}(z)\right)^{-1}:=\hat{C}\left(z^{-1} I-\hat{A}\right)^{-1} \hat{B}+\hat{D} .
$$

Since existence and boundedness of $\left(I-\hat{G}_{N} \hat{\Delta}\right)^{-1}$ imply existence of (14) for all $|z| \leq 1$ (Lemma 4.2 of 10)), $\left(z^{-1} I-\hat{A}\right)^{-1}$ exists for all $|z| \leq 1$, in other words $\rho(\hat{A})<1$. The condition $\rho(\hat{A})<1$ (from Theorem 4.2 of 8$)$ ) implies

$$
\left\|\hat{A}^{k}\right\| \leq M_{8} \beta_{6}^{k} .
$$

We rewrite $(12)$ as

$$
\hat{v}=\left(I-\hat{G}_{N} \hat{\Delta}\right)^{-1}(\hat{p}+\hat{q}) .
$$

Using the notation $(\hat{A}, \hat{B}, \hat{C}, \hat{D})$, we have

$$
\begin{gathered}
\hat{v}_{k}=\sum_{i=0}^{k-1} \hat{C} \hat{A}^{k-1-i} \hat{B}\left(\hat{p}_{i}+\hat{q}_{i}\right)+\hat{D}\left(\hat{p}_{k}+\hat{q}_{k}\right), \\
k \geq 1
\end{gathered}
$$

Noting $\left\|\hat{p}_{i}+\hat{q}_{i}\right\|_{L_{n}^{2}[0, \tau)} \leq M_{9} \beta_{7}^{i}\left(\left\|\hat{X}_{0}\right\|_{2}+\left\|\hat{Y}_{0}\right\|\right), \quad i \geq 0$, and using (15), we can obtain (10) with the similar way to (11).

Lemma 2 guarantees the exponential stability of the system $\hat{G}_{N}$ with $\hat{\Delta}$ for any initial conditions $\hat{X}_{0}$ and $\hat{Y}_{0}$ under some condition. Hence Lemma 2 guarantees stability of $\hat{G}_{N}$ for the initial conditions in Lemma 1. Since elements of $\hat{X}_{k}$ coincides with the sampled-states of the system $G$ with the initial conditions in Lemma 1, Lemma 2 implies stability of $G$. Based on this observation, we are ready to state the main result of this paper.

Theorem 1. If $\rho\left(\hat{A}_{N}\right)<1$ and $\left\|\hat{G}_{N 11}\right\|_{\infty}<1$, then the system $G$ is exponentially stable.

Proof. As stated in the above, $\|x(k \tau)\|_{2}$ and $\|x(k \tau+t)-x(k \tau)\|_{L_{n}^{2}[0, \tau)}$ of $G$ are exponentially convergent if $\rho\left(\hat{A}_{N}\right)<1$ and $\left\|\hat{G}_{N 11}\right\|_{\infty}<1$ from Lemmas 1 and 2. Since (8) consists of bounded functions of $t$, $\|x(k \tau+t)\|_{2}$ is also exponentially convergent.

The condition $\rho\left(\hat{A}_{N}\right)<1$ is concerned with the stability of $\hat{G}_{N 22}$ and under the condition $\left\|\hat{G}_{N 11}\right\|_{\infty}<1$ the stability of $\hat{G}_{N 22}$ implies the stability of $G$. In order to use Theorem 1 , it is necessary to compute $\left\|\hat{G}_{N 11}\right\|_{\infty}$, which cannot be directly done since the input and output spaces of $\hat{G}_{N 11}$ are infinite dimensional. This condition, however, can be transformed into an $H_{\infty}$ norm test for a discrete-time system with the help of the results in 7) and 9).

Lemma 3. Let us assume $\left\|\hat{D}_{11}\right\|_{\infty}<1$, then $\left\|\hat{G}_{N 11}\right\|_{\infty}<1$ if and only if $\left\|\bar{G}_{N}\right\|_{\infty}<1$. The realization of $\bar{G}_{N}$ is given by

$$
\bar{G}_{N}=\left[\begin{array}{c|c}
\bar{A}_{N} & \bar{B} \\
\hline \bar{C} & 0
\end{array}\right]:\left[l_{R^{b}}\right] \rightarrow\left[l_{R^{c}}\right]
$$

where $\bar{A}_{N} \in R^{n(N+1) \times n(N+1)}, \bar{B} \in R^{n(N+1) \times n}$, and $\bar{C} \in R^{n \times n(N+1)}$ are given by

$$
\bar{A}_{N}=\left[\begin{array}{ccccc}
S_{1} & & & & S_{2} \\
I & & & \\
& I & & \\
& & \ddots & \\
& & & I
\end{array}\right]
$$

$$
\bar{B}=T_{B^{*}}\left[\begin{array}{c}
\Sigma_{b}^{\frac{1}{2}} \\
0
\end{array}\right], \quad \bar{C}=\left[\begin{array}{ll}
\Sigma_{c}^{\frac{1}{2}} & 0
\end{array}\right] T_{C}
$$


$T_{B^{*}}\left[\begin{array}{ll}\Sigma_{b} & \\ & 0\end{array}\right] T_{B}=\left[\begin{array}{ll}S_{3} & \\ & \end{array}\right]$,
$T_{C^{*}}\left[\begin{array}{ll}\Sigma_{c} & \\ & 0\end{array}\right] T_{C}=\left[\begin{array}{ll}S_{4} & S_{5} \\ & \\ S_{6} & S_{7}\end{array}\right]$,

$S_{1}=\Gamma_{22}-\Gamma_{21} \Gamma_{11}^{-1} \Gamma_{12}-\Gamma_{21} \Gamma_{11}^{-1} \Phi_{11}+\Phi_{21}$,

$S_{2}=\left(\Phi_{22}-\Gamma_{21} \Gamma_{11}^{-1} \Phi_{12}\right) A_{1}$,

$S_{3}=\Gamma_{21} \Gamma_{11}^{-1}$,

$S_{4}=-\Gamma_{11}^{-1} \Gamma_{12}-\Gamma_{11}^{-1} \Phi_{11}+\Phi_{21} \Gamma_{11}^{-1} \Gamma_{12}-\Phi_{22}$ $+\Phi_{21} \Gamma_{11}^{-1} \Phi_{11}-\Omega_{21}+\tau I$,

$S_{5}=\left(-\Gamma_{11}^{-1} \Phi_{12}+\Phi_{21} \Gamma_{11}^{-1} \Phi_{12}-\Omega_{22}\right) A_{1}$,

$S_{6}=A_{1}^{\prime}\left(\Phi_{12}-\Phi_{11} \Gamma_{11}^{-1} \Gamma_{12}-\Phi_{11} \Gamma_{11}^{-1} \Phi_{11}+\Omega_{11}\right)$,

$S_{7}=A_{1}^{\prime}\left(\Omega_{12}-\Phi_{11} \Gamma_{11}^{-1} \Phi_{12}\right) A_{1}$,

$\Gamma(t)=\exp \left(\left[\begin{array}{cc}-A_{0}^{\prime} & -I \\ A_{1} A_{1}^{\prime} & A_{0}\end{array}\right] t\right)$

$\Phi(t)=\int_{0}^{t} \Gamma(r) d r, \Omega(t)=\int_{0}^{t} \int_{0}^{s} \Gamma(r) d r d s$.

In the above equations, $T_{B}, \Sigma_{b}, T_{C}$, and $\Sigma_{c}$ come from the symmetric decompositions of their corresponding righthand sides, and $\Psi, \Gamma, \Phi$, and $\Omega$ denote $\Psi(\tau), \Gamma(\tau), \Phi(\tau)$, and $\Omega(\tau)$, respectively. All unspecified entries in the above matrices are zero matrices with appropriate sizes.

Proof. Although the calculation needed to derive $\bar{G}_{N}$ is slightly more complicated, the proof is a direct application of Theorem 6 in 9 ).

The condition $\left\|\hat{D}_{11}\right\|_{\infty}<1$ can be checked using the algorithms in 9) and 12). Therefore using Lemma 3 we can now test the stability of $G$. We do this for the following simple example ${ }^{3)}$ :

$$
\dot{x}(t)=\left[\begin{array}{cc}
-2 & -1 \\
0 & -2
\end{array}\right] x(t)+\gamma\left[\begin{array}{ll}
0 & 1.2 \\
1 & 1.1
\end{array}\right] x(t-0.3) .
$$

The ranges of $\gamma$, under which the stability conditions of Theorem 1 assure the stability of the above system, are listed in Table 1. A delay-independent stability condition in 3) and the exact stability condition computed using the algorithm in 2) are also listed for comparison. We note that wider range of $\gamma$ implies that the corresponding stability condition is less conservative.

It can be conjectured from Table 1 that the stability condition of Theorem 1 converges to the exact stability condition as $N$ increases. This is indeed so and is proved in the next two lemmas.

Lemma 4. $\left\|\hat{G}_{N 11}\right\|_{\infty} \rightarrow 0$ as $N \rightarrow \infty$.
Table 1 Stability range of stability conditions

\begin{tabular}{|c|c|}
\hline & Bounds of $\gamma$ \\
\hline Stability condition in 3$)$ & $-1.297<\gamma<1.297$ \\
\hline Theorem 1 $(N=1)$ & $-1.468<\gamma<1.392$ \\
\hline Theorem 1 $(N=2)$ & $-2.140<\gamma<1.392$ \\
\hline Theorem 1 $(N=3)$ & $-2.392<\gamma<1.392$ \\
\hline Exact stability condition 2$)$ & $-2.392<\gamma<1.392$ \\
\hline
\end{tabular}

Proof. Since $\tau=h / N, \tau \rightarrow 0$ as $N \rightarrow \infty$. To prove $\left\|\hat{G}_{N 11}\right\|_{\infty} \rightarrow 0$ as $N \rightarrow \infty$, it suffices to show that $\hat{C}_{11} \rightarrow 0$ and $\left\|\hat{D}_{11}\right\|_{\infty} \rightarrow 0$ as $N \rightarrow \infty$. The fact $\hat{C}_{11} \rightarrow 0$ as $N \rightarrow \infty$ is obvious. Note that $\hat{D}_{11}: l_{L_{n}^{2}[0, \tau)} \rightarrow l_{L_{n}^{2}[0, \tau)}$ can be denoted by $p(t)=\int_{0}^{t} e^{A_{0}(t-s)} A_{1} q(s) d s$, then

$$
\begin{aligned}
& \|p\|_{L^{2}}^{2}[0, \tau) \\
& \quad=\int_{0}^{\tau}\left\|\int_{0}^{t} e^{A_{0}(t-s)} A_{1} q(s) d s\right\|_{2}^{2} d t \\
& \quad \leq \int_{0}^{\tau} \int_{0}^{t} \bar{\sigma}^{2}\left(e^{A_{0}(t-s)} A_{1}\right) d s\|q\|_{L^{2}[0, \tau)}^{2} d t \\
& \quad \leq \int_{0}^{\tau} \int_{0}^{\tau} \bar{\sigma}^{2}\left(e^{A_{0}(t-s)} A_{1}\right) d s d t\|q\|_{L^{2}[0, \tau)}^{2},
\end{aligned}
$$

where $\sigma(M)$ denotes the maximum singular value of $M$. The above relations imply that $\left\|\hat{D}_{11}\right\|_{\infty} \rightarrow 0$ as $\tau \rightarrow 0$, or equivalently as $N \rightarrow \infty$.

Lemma 5. If $G$ is stable, then $\rho\left(\hat{A}_{N}\right)<1$ as $N \rightarrow \infty$. If $G$ is unstable, then $\rho\left(\hat{A}_{N}\right)>1$ as $N \rightarrow \infty$.

Proof. The proof is a slight extension of the result in 14). We will assume the system $G$ has no imaginary pole and $h=1$ for simplicity. First we will show that the eigenvalues of $\hat{A}_{N}$ approach the solutions of (2) as $N \rightarrow \infty$. The eigenvalues of $\hat{A}_{N}$ are the solutions of the following equation.

$$
\begin{aligned}
& \operatorname{det}\left(z I-\hat{A}_{N}\right)= \\
& \operatorname{det}\left(z^{N+1} I-z^{N} e^{A_{0} \frac{1}{N}}-\int_{0}^{\frac{1}{N}} e^{A_{0} r} A_{1} d r\right)=0 .
\end{aligned}
$$

If we write the solutions of (18) as $z_{1}, \cdots, z_{n \times(N+1)}$, then each solution $z_{i}, \quad 1 \leq i \leq n \times(N+1)$, can be expressed by $z_{i}=e^{\left(\mu_{i} / N\right)}$ for some $\mu_{i} \in C$. If we apply the following relations into (18),

$$
\begin{array}{ccc}
e^{\frac{1}{N-1} A_{0}} & = & I+\frac{1}{N-1} A_{0}+O\left(N^{2}\right), \\
\int_{0}^{\frac{1}{N-1}} e^{A_{0} r} A_{1} d r & = & \frac{1}{N-1} A_{1}+O\left(N^{2}\right),
\end{array}
$$

we get the following:

$$
\operatorname{det}\left(\mu_{i} I-A_{0}-A_{1} e^{\mu_{i}}+O(N)\right)=0 .
$$

The equation $P=Q+O(N)$ should be understood that $\lim _{N \rightarrow \infty} \bar{\sigma}(P-Q) / N \rightarrow 0$. Since $\operatorname{det}(\cdot)$ is a continuous operator and the quasipolynomial (2) is continuous ${ }^{13)}$, the following are satisfied ( $s$ and $\mu_{i}$ denote the solutions of (2) and (18), respectively).

(i) For every real number $\varepsilon>0$ and for every $\mu_{i}$, there exists an integer $N_{1}(\varepsilon)$ such that $\left|\mu_{i}-s\right|<\varepsilon$ for some $s$ if $N>N_{1}(\varepsilon)$.

(ii) For every real number $\varepsilon>0$ and for every $s$, there 
exists an integer $N_{2}(\varepsilon)$ such that $\left|s-\mu_{i}\right|<\varepsilon$ for some $\mu_{i}$ if $N>N_{2}(\varepsilon)$.

If $G$ is stable, there holds $\max \{\operatorname{Re}(s)\}=\delta<0$. The fact (i) implies that there exists $N_{1}\left(-\frac{\delta}{2}\right)$ such that $\left|\mu_{i}-s\right|<-\frac{\delta}{2}, \forall \mu_{i}$, and consequently, $\max \left\{\operatorname{Re}\left(\mu_{i}\right)\right\}<$ $\max \{\operatorname{Re}(s)\}-\frac{\delta}{2}<\frac{\delta}{2}<0$. Therefore $\rho\left(\hat{A}_{N}\right)<1$ as $N \rightarrow \infty$.

Similarly if $G$ is unstable, then there exists at least one $s$ such that $\operatorname{Re}(s)>\delta>0$. The fact (ii) implies that there exists $\mu_{i}$ such that $\operatorname{Re}\left(\mu_{i}\right)>0$ as $N \rightarrow \infty$. Therefore $\rho\left(\hat{A}_{N}\right)>1$ as $N \rightarrow \infty$.

We can see the computational burden to check conditions of Lemma 3 is proportional to the $N$ (see the dimensions of $\bar{A}_{N}$ ). Hence we cannot allow $N \rightarrow \infty$, although the condition based on larger $N$ is less conservative. Therefore, we have to choose an appropriate $N$, taking a tradeoff between conservatism and computational burden.

\section{Conclusion}

In this paper, a new delay-dependent stability condition was derived. This stability condition is based on discretization of state-delayed systems. If the derived stability condition is satisfied, the stability of the finitedimensional part of the discretized system can imply the original stability of state-delayed systems. This stabilityguaranteed discretization is a new approach to stability analysis of state-delayed systems and is the main contribution of this paper. The stability condition is easily applicable to synthesis problems and we are currently working on synthesis problems based on the derived stability condition.

\section{Acknowledgment}

The authors wish to thank the reviewers for their detailed comments and helpful suggestions for the revision.

\section{References}

1) M. C. Delfour, C. McCalla, and S. K. Mitter: Stability and the Infinite-time Quadratic Cost Problem for Linear Hereditary Differential Systems, SIAM J. Control, 13-1, 48/88 (1975)

2) A. Manitius, H. Tran, G. Payre, and R. Roy: Computation of Eigenvalues Associated with Functional Differential Equations, SIAM J. Sci. Stat. Comput., 8-3, 222/247 (1987)

3) H. Trinh and M. Aldeen: On the Stability of Linear Systems with Delayed Perturbations, IEEE Trans. Automat. Contr., 39-9, 1948/1951 (1994)

4) J. Chen, D. Xu, and B. Shafai: On Sufficient Conditions for Stability Independent of Delay, IEEE Trans. Automat. Contr., 40-9, 1675/1680 (1995)

5) T. Mori and H. Kokame: Stability of $\dot{x}(t)=A x(t)+B x(t-$ $\tau)$, IEEE Trans. Automat. Contr., 34-4, 460/462 (1989)
6) J.-H. Su, I.-K. Fong, and C.-L. Tseng: Stability Analysis of Linear Systems with Time Delay, IEEE Trans. Automat. Contr., 39-6, 1341/1344 (1994)

7) P. T. Kabamba and S. Hara: Worst-Case Analysis and Design of Sampled-Data Control Systems, IEEE Trans. Automat. Contr., 38-9, 1337/1357 (1993)

8) Y. Yamamoto: A Function Space Approach to Sampled Data Control Systems and Tracking Problems, IEEE Trans. Automat. Contr., 39-4, 703/713 (1994)

9) B. Bamieh and J. B. Pearson, Jr.: A General Framework for Linear Periodic Systems with Application to $H_{\infty}$ Sampled-data Control, IEEE Trans. Automat. Contr., 374, 418/435 (1992)

10) G. E. Dullerud: Control of Uncertain Sampled-Data Systems, PhD thesis, University of Cambridge (1994)

11) M. Green and D. J. N. Limebeer: Linear Robust Control, Prentice-Hall (1995)

12) C. Foias, A. Tannenbaum, and G. Zames: Some Explicit Formulae for the Singular Values of Certain Hankel Operators with Factorizable Symbol, SIAM J. Math. Anal., 19-5, 1081/1089 (1988)

13) K. L. Cooke and J. M. Ferreira: Stability Conditions for Linear Retarded Functional Differential Equations, J. Math. Ana. \& Appli., 96, 480/504 (1983)

14) J. Hale: Theory of Functional Differential Equations, Springer-Verlag (1977)

15) A. W. Naylor and G. R. Sell: Linear Operator Theory in Engineering and Science, Springer-Verlag (1982)

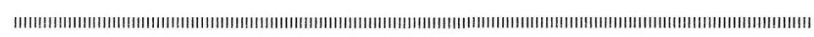

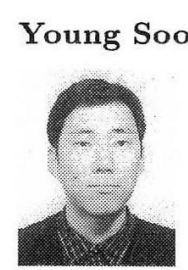

Sur (Member)

He received the B.E. and M.E. degrees from Seoul National University, Korea, in 1990 and 1992, respectively and the Ph.D. degree in 1997 from the University of Tokyo. He is now with National Institute of Materials and Chemical Research. His research interests include time delay system control and robust control.

\section{Seiichi Shin (Member)}

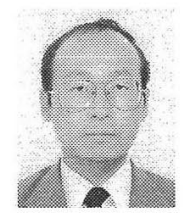

He was born on May 51954 in Oita Prefecture. He received Bachelor, Master, and Doctor Degrees of Engineering all from the University of Tokyo, in 1978, 1980, and 1987 respectively. He is now an associate professor of Graduate School of Engineering, the University of Tokyo. He received SICE paper awards in 1991 and 1993. He also received the award with Takeda Prize in 1992. His main interest is a research of theory and application of control. $\mathrm{He}$ is a councilor of JSIAM, and a member of ISCIE, IEEJ, JSME, IEEE, and so on.

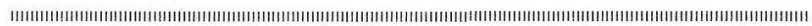

